\title{
Chromosomal instability triggers cell death via local signalling through the innate immune receptor Toll
}

\author{
Dawei Liu ${ }^{1}$, Zeeshan Shaukat ${ }^{1}$, Robert B. Saint ${ }^{1}$ and Stephen L. Gregory ${ }^{1}$ \\ ${ }^{1}$ School of Biological Sciences, University of Adelaide, Adelaide, Australia \\ Correspondence to: Stephen L. Gregory, email: stephen.gregory@adelaide.edu.au \\ Keywords: chromosomal instability, innate immune response, Drosophila, TNFa, TLRs, Immunology Section, Immunity, Immune re- \\ sponse \\ Received: July 13,2015 Accepted: September 08, $2015 \quad$ Published: October 10, 2015
}

This is an open-access article distributed under the terms of the Creative Commons Attribution License, which permits unrestricted use, distribution, and reproduction in any medium, provided the original author and source are credited.

\section{ABSTRACT}

Chromosomal instability (CIN) is a hallmark of cancer and has been implicated in cancer initiation, progression and the development of resistance to traditional cancer therapy. Here we identify a new property of CIN cells, showing that inducing CIN in proliferating Drosophila larval tissue leads to the activation of innate immune signalling in CIN cells. Manipulation of this immune pathway strongly affects the survival of CIN cells, primarily via JNK, which responds to both Toll and TNF $\alpha$ /Eiger. This pathway also activates Mmp1, which recruits hemocytes to the CIN tissue to provide local amplification of the immune response that is needed for effective elimination of CIN cells.

\section{INTRODUCTION}

Chromosomal Instability (CIN) refers to a state in which cells have an increased rate of gain or loss of whole chromosomes or chromosomal pieces [1]. Several causes of CIN have been identified, and include dysfunction of the spindle assembly checkpoint, centrosomes, DNA replication or cohesion, leading to defects in chromosome segregation during mitosis $[1,2]$. CIN is a defining feature of most human solid cancers (e.g. $85 \%$ of sporadic colorectal cancers) and is positively correlated with both drug resistance and poor prognosis [3]. Somatic cells with CIN can initiate the process of tumorigenesis [4], and CIN is responsible for the generation of cells with varied genetic backgrounds, out of which drug resistance can develop. This is thought to contribute to relapses following traditional cancer therapies that appear to initially succeed: the therapy creates selection pressure on cancer cells which drives rapid genetic evolution as CIN generates diverse cells from which those with a selective advantage and drug resistance can arise [5].

In this context, the role of the immune system is a double-edged sword during tumorigenesis [6]. On one hand, tumor-related inflammation is thought to foster tumorigenesis by supplying molecules into the tumour micro-environment that promote angiogenesis, resistance to apoptosis, and metastasis of malignant cells [7]. On the other hand, the immune system can detect and eliminate incipient cancer cells: there is good evidence for cancer immune surveillance. For example, immunocompromised mice that lack mature lymphocytes show a higher frequency of spontaneous tumorigenesis by the age of 14-16 months [8]. The frequency of carcinogen-induced tumorigenesis is also much higher in immunocompromised mice than in immunocompetent controls [9]. Furthermore, clinical evidence shows that at least for some kinds of tumours, increased infiltration with activated $\mathrm{T}$ cells is correlated with a better prognosis $[10$, 11]. Overall, the capability of cancer cells to circumvent attack by the immune system has been recognized as a hallmark of cancer [12].

Chromosomal instability represents a striking difference between the tumour and stromal cells, which do not normally have CIN. Consequently, CIN represents an excellent immune target if it can be recognized. Although the immune system has been reported to be activated by DNA damage [13] and tissue dysplasia [14], little is known about in vivo responses to CIN. While screening for genes that are required for the death of CIN cells in vivo, we identified several immune signalling genes. We found that the induction of CIN not only activates a systemic response from immune tissues, but also triggers a local immune reaction in proliferating epithelial cells. Manipulation of immune signalling strongly affects the 
fate of these CIN cells. Altogether, our results showed that the immune system can detect and respond to CIN, and represents a critical feedback loop that is necessary to ensure the removal of defective cells that are a threat to the organism.
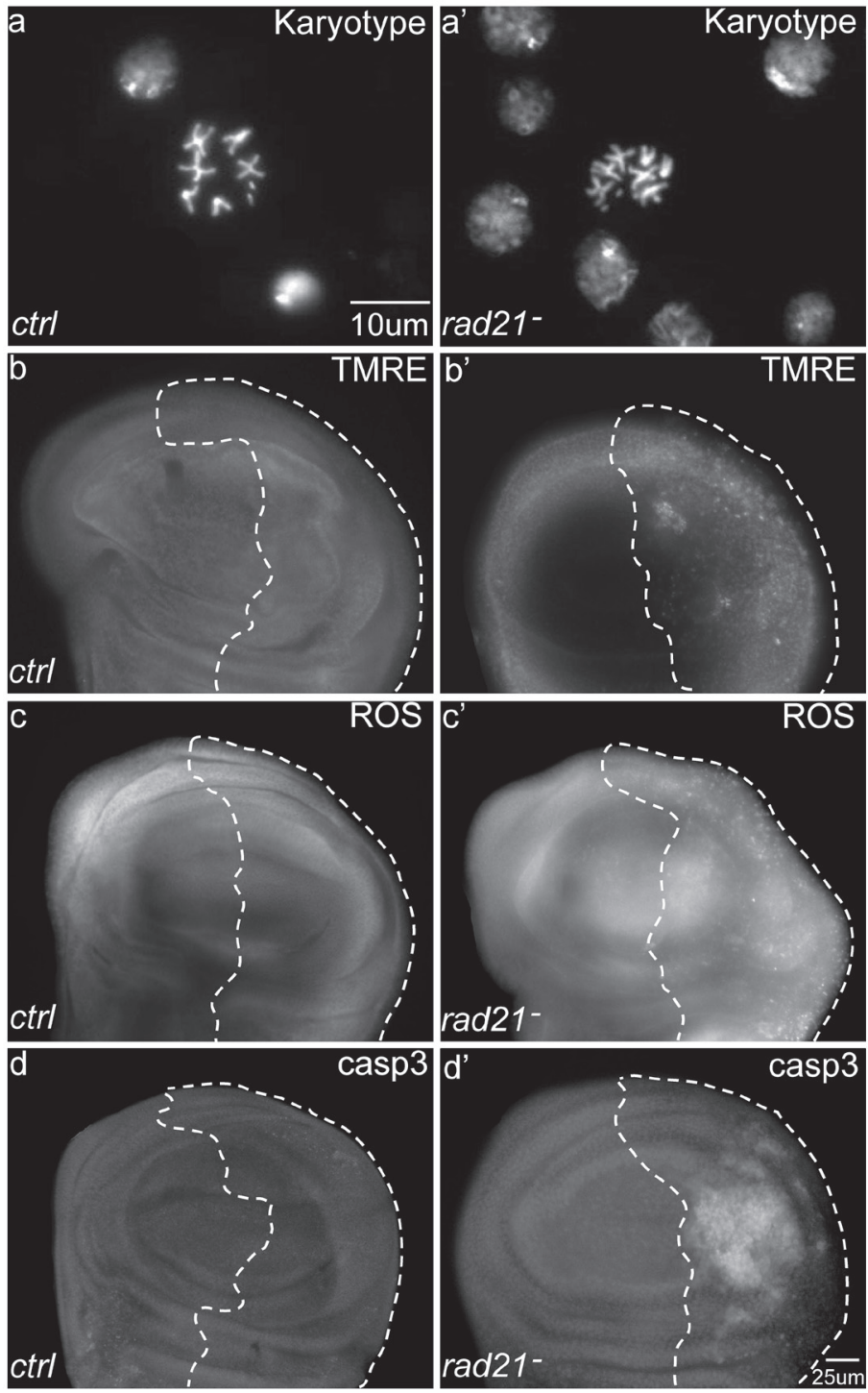

Figure 1: The effects of Chromosomal Instability (CIN) induced by Rad21 depletion on larval wing discs. CIN was induced in the engrailed expressing posterior region of the wing discs as shown by the dotted line; the rest of each disc was wild type. (a, a') DNA stains of metaphase cells to show the karyotype. (a) A normal karyotype. (a') Karyotype from a wing disc cell with induced CIN (engrailed $>$ Gal4, UAS-rad2 $1^{\mathrm{RNAi}} U A S$-Dicer2) showing a chromosome gain. Aneuploidy rates were quantified in Fig S1. (b, b') TMRE staining of third instar larval wing discs. Tissue with induced CIN (engrailed $>$ Gal4, UAS-rad2 $1^{\text {RNAi }}$ UAS-Dicer2) showed increased mitochondrial membrane potential (b') compared to the negative control (b). (c, $\mathbf{c}^{\prime}$ ) CellRox staining of third instar larval wing discs. CIN cells showed increased oxidative stress ( $\left.\mathbf{c}^{\prime}\right)$ compared to the negative control (c). (d, d') Anti-cleaved caspase 3 antibody staining of third instar larval wing discs. CIN tissue showed an increased level of apoptosis (d') compared to the negative control (d). 
a repair response from the JNK pathway $[16,17]$. In order to generate higher levels of CIN and to confirm that these CIN phenotypes were not specific to mad2 knockdown, we created another inducible-CIN model. We knocked down rad21, a cohesin that regulates sister chromatid separation during cell division $[18,19]$. While $\operatorname{Rad} 21$ mutation is not common in advanced cancers, its depletion results in CIN in vertebrates [20]. Co-expressing Dicer2 to enhance the RNAi knockdown of rad 21 in proliferating wing imaginal disc cells resulted in aneuploidy in $46 \%$ of metaphase cells, indicating a relatively high rate of CIN (Figure 1a' and Figure S1). To avoid missing cells that may have died from aneuploidy and been cleared, we blocked apoptosis by overexpression of p35 and in this case saw that around
$70 \%$ of metaphase cells were aneuploid (Figure S1b). CIN induced by rad21 depletion led to an increase in the level of TMRE staining, indicating elevated mitochondrial activity (Figure 1 $b^{\prime}$ ). As expected, this was accompanied by an increased level of oxidative stress (Figure 1c') and widespread cell death (Figure 1d', Figure S1). These effects were consistent with, but stronger than the effects of mad2 knockdown [16, 21]. We found that we could similarly increase the rate of aneuploidy and cell death in the $\operatorname{mad} 2$ model by using temperature to increase the RNAi expression level or by blocking apoptosis (Figure S1b). These results indicated that chromosomal instability generated by disparate means resulted in mitochondrial dysfunction and oxidative stress. Using strong depletion

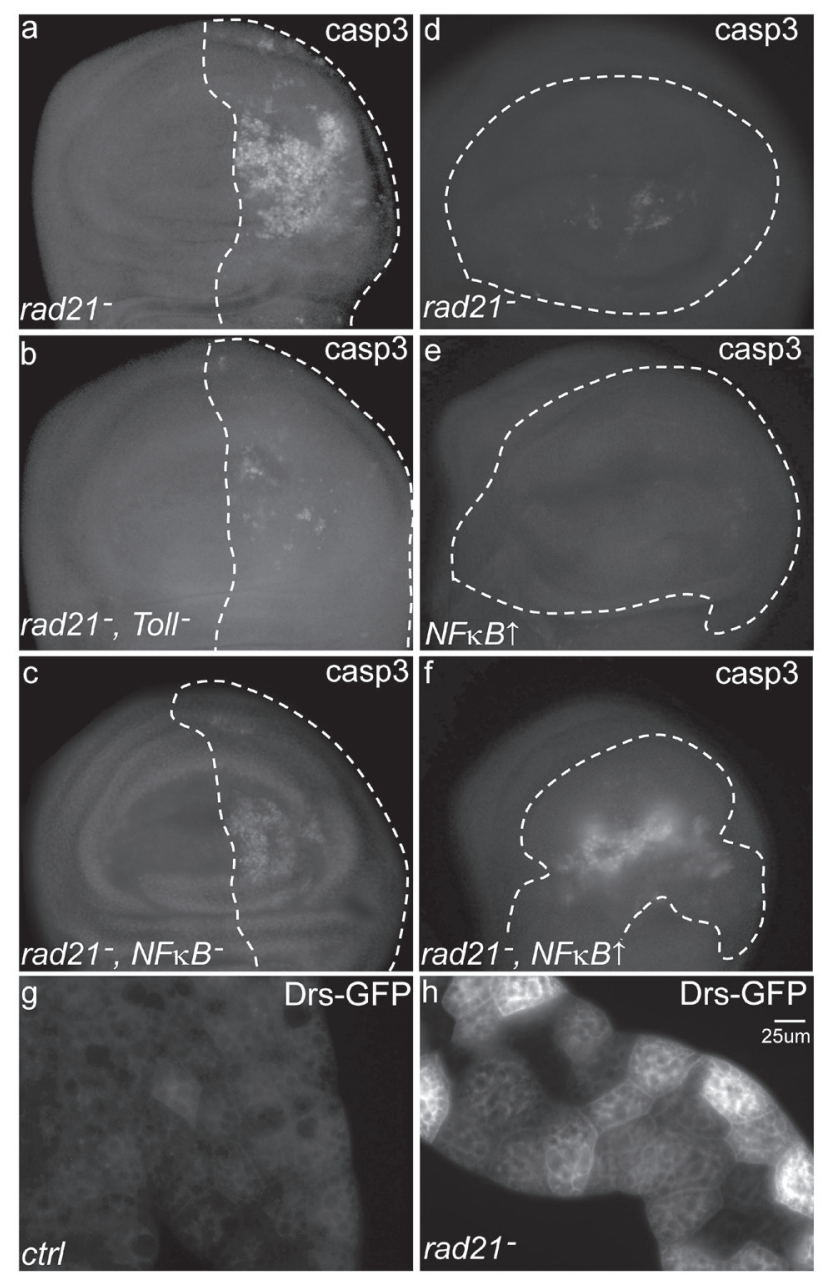

Figure 2 The effect of Toll pathway knock down or activation on CIN cell apoptosis. Anti-cleaved caspase 3 antibody staining shows apoptosis in third instar larval wing discs with CIN induced by depletion of Rad21 (UAS-rad2 $\left.1^{\mathrm{RNAi}} U A S-D i c e r 2\right)$. (a, b and $\left.\mathbf{c}\right) \mathrm{CIN}$ and Toll pathway gene double knockdowns were induced in the engrailed region (driven by engrailed $>$ Gal4) of the wing discs as shown by the dotted line; the remainder of each disc was wild type. Knocking down Toll (b) or NFkB (dorsal) (c) significantly reduced the level of apoptosis in CIN cells (a). (d, e and f) CIN and gene overexpression were induced in the dotted-lined region (driven by MS1096 $>$ Gal4) while the rest of each disc was wild type. Activation of the Toll pathway by NFkB (dorsal) over-expression caused little apoptosis in normal cells (e), but greatly increased the level of apoptosis in CIN cells (f). Note that the level of cell death induced by Rad21-RNAi was lower using the MS1096 driver (d-f) than with the engrailed driver (a-c), allowing detection of CIN enhancement without killing the animal. Quantitation for these results is shown in Fig S2. The innate immune response from the primary immune tissue, the fat body, was detected by visualizing levels of a GFP-tagged antimicrobial peptide (Drosomycin-GFP) in the larval fat body (g, h). Wild type larvae expressed low levels of Drosomycin-GFP (g), but this level was increased in animals in which CIN had been induced in a range of tissues including the wing, epidermis, gut and fat body (h, engrailed $>$ Gal4, UAS-rad2 $1^{\text {RNAi } U A S-D i c e r 2) . ~}$ 
of $\operatorname{rad} 21$ or $\operatorname{mad} 2$ we were able to generate high levels of instability making many cells inviable.

\section{Depletion of the toll pathway rescues lethality and apoptosis caused by chromosomal instability}

Having generated models in which high levels of CIN caused cell death, we were in a position to identify mechanisms that might be mutated in CIN cells (such as tumours) to improve their tolerance of this detrimental phenotype. Ubiquitous knockdown of mad2 in Drosophila resulted in no adult survivors at $30^{\circ} \mathrm{C}$, so we tested candidate gene knockdowns to identify any that could rescue this CIN lethality. While testing candidates involved in a variety of cellular processes, we found that knockdown of five Drosophila innate immune response genes from the Toll pathway could rescue the lethality in CIN flies (Toll, dorsal, spatzle, cactus, and pelle). These genes are part of a conserved signalling pathway that regulates patterning during early development and subsequently is used to mediate innate immune responses
[22]. We next carried out cell death assays to examine whether the increased viability observed was due to a reduction in cell death when the Toll pathway was depleted in CIN cells. Knockdown of Toll or the NFкB homolog dorsal in CIN cells significantly reduced the rate of cell death as detected by Acridine Orange incorporation and anti-cleaved-caspase 3 staining for apoptosis (Figure $2 \mathrm{a}-2 \mathrm{c}$ and Figure S2). We confirmed that the level of knockdown of Mad2 was not decreased when we also knocked down Toll (Figure S1), excluding the possibility of Gal4 titration. Furthermore, we found that simulating Toll pathway activation by $\mathrm{NF} \kappa \mathrm{B} /$ dorsal overexpression greatly increased the level of apoptosis in CIN cells (Figure 2f) but had a limited effect on normal cells (Figure 2e). These results suggested that local activation of Toll pathway in CIN tissue is needed for the appropriate cell death response to high levels of CIN.

\section{CIN leads to an immune response}

The Toll transmembrane receptor has been implicated in the induction of innate immune responses
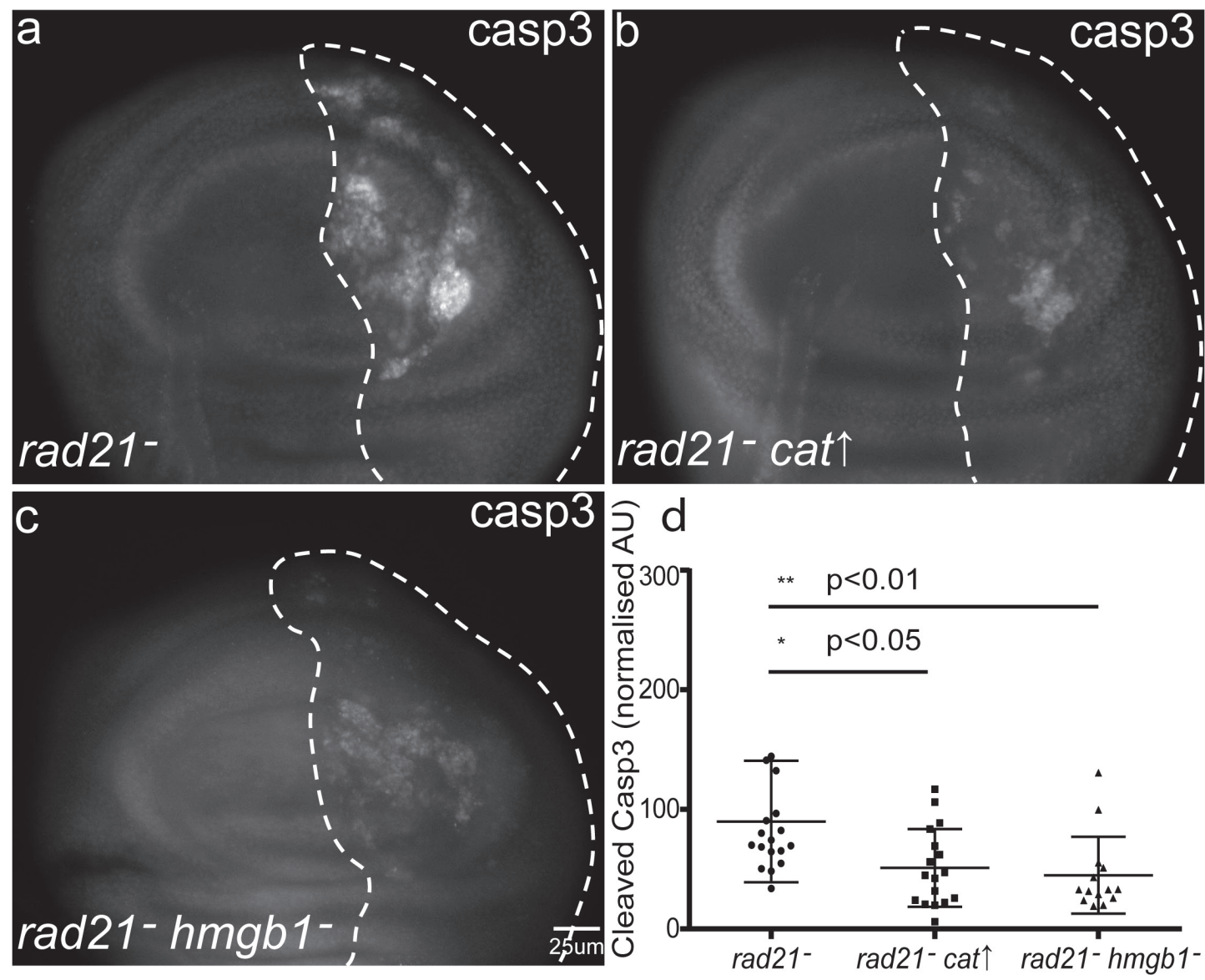

Figure 3: The role of reactive oxygen species (ROS) in determining the fate of CIN cells. Anti-cleaved caspase 3 antibody was used to detect apoptosis in third instar larval wing discs with CIN induced in the posterior (dotted) region (engrailed $>$ Gal4, UAS$\operatorname{rad} 21^{\mathrm{RNAi}} U A S$-Dicer2) (a). Overexpression of Catalase to reduce oxidative stress (b) or knocking down the redox-sensitive damage marker HMGB1 (c) significantly reduced the level of apoptosis in CIN cells. (d) Quantification of the cleaved caspase 3 staining. In all cases $\mathrm{n} \geq 10$ and the error bars show 95\% confidence intervals. The $p$ values were calculated using two-tailed t-tests with Welch's correction. 
[23], so we hypothesized that the immune system could be activated in response to CIN. Drosomycin is an antimicrobial peptide gene that is a direct transcriptional target of Toll/NFkB signalling [24], so we used a Drosomycin-GFP reporter to detect its expression in the primary immune secretory tissue (fat body) of CIN larvae (Figure 2g, 2h). We observed a strong up-regulation of Drosomycin-GFP signal in 0 out of 12 control larvae and 11 out of 12 larvae with induced CIN. Together, these results suggest that induction of CIN leads to activation of the larval innate immune response. We also performed immunostaining against Dorsal and Relish, NF $\kappa$ B mediators of the Drosophila innate immune system downstream of Toll [25]. We found elevated levels of Dorsal in the cytoplasm of CIN wing disc cells (Figure S3). We observed a barely detectable increase in Relish (downstream of IMD) in CIN cells, even when using p35 to block apoptosis [26] and retain highly aneuploid cells
(Figure S3).

\section{The immune system responds to reactive oxygen species}

Having found that the induction of CIN triggers an immune response we wished to understand what aspect of CIN cell biology is detected by the immune system. Reactive Oxygen Species (ROS) are known to activate both sterile and infectious inflammatory responses [27]. We have previously shown that CIN cells generate elevated levels of ROS [16], so we hypothesized that ROS might be a trigger. We found that over-expression of Catalase, which decreases ROS levels by converting $\mathrm{H}_{2} \mathrm{O}_{2}$ into $\mathrm{H}_{2} \mathrm{O}$, significantly rescues the apoptosis observed in CIN cells (Figure $3 b$ and Figure S4a-b). Knocking down the Drosophila ortholog of HMGB1 (Dsp1), a ROSresponsive effector of immune activation in vertebrates
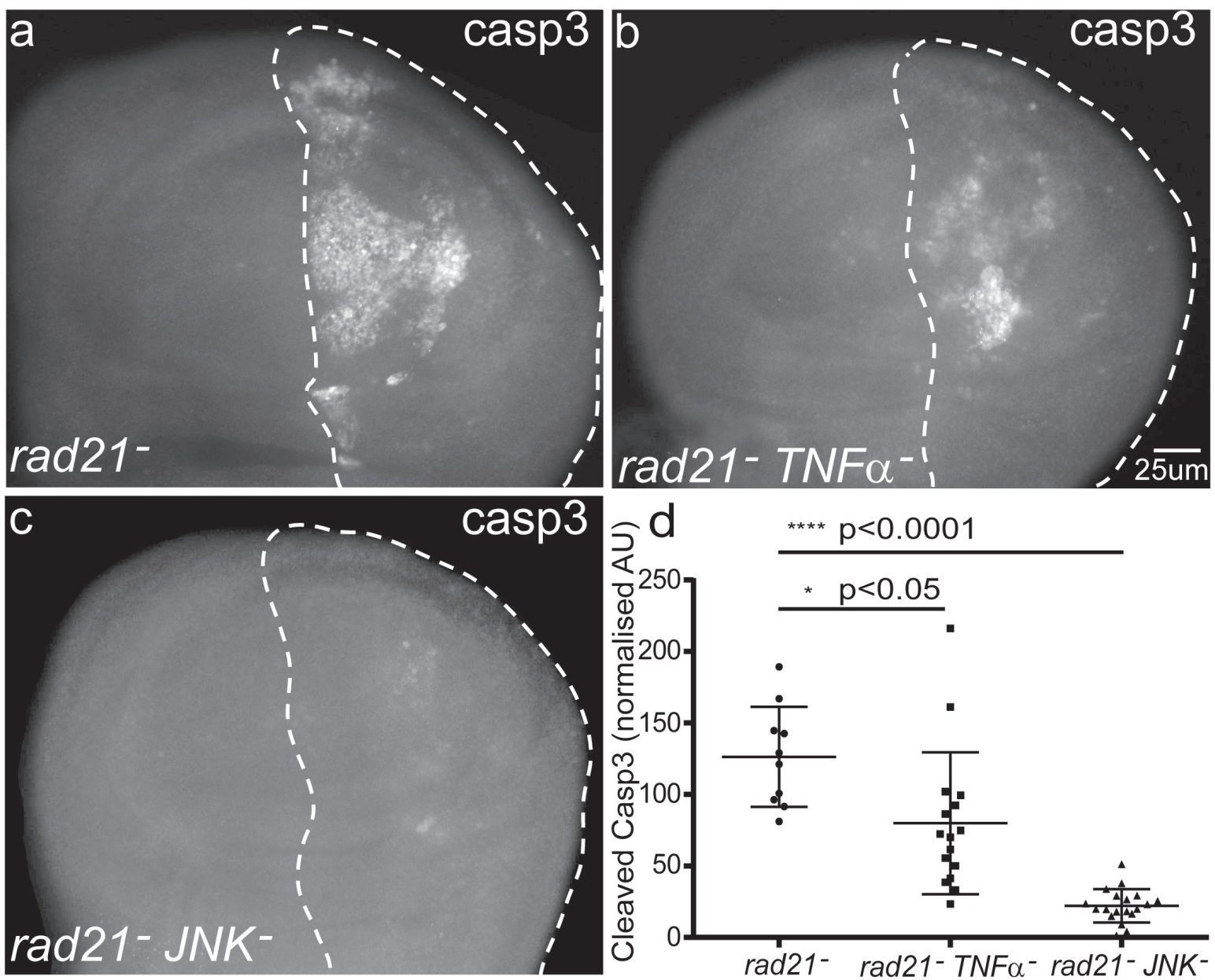

Figure 4: The effect of blocking TNFa signalling by depletion of Eiger or JNK, on the fate of CIN cells. (a-d) Anticleaved caspase 3 antibody was used to detect apoptosis in third instar larval wing discs with CIN induced in the posterior (dotted) region (engrailed $>$ Gal4, UAS-rad2 $1^{\mathrm{RNAi}} U A S$-Dicer2). Knocking down either TNF $\alpha$ (UAS-eiger ${ }^{\mathrm{RNAi}}$ (b) or JNK (UAS-bsk ${ }^{\mathrm{RNAi}}$ ) (c) significantly reduced the rate of apoptosis in CIN cells. In these experiments TNF $\alpha$ production by immune cells such as circulating hemocytes was not altered; the knockdown was restricted to engrailed-expressing tissues such as the imaginal discs. Panel (d) shows quantification of the cleaved caspase 3 staining. In all cases $\mathrm{n} \geq 10$ and the error bars show $95 \%$ confidence intervals. The $p$ values were calculated using twotailed t-tests with Welch's correction. 
$[27,28]$ also rescued the apoptosis phenotype in CIN cells (Figure 3c). These results suggest a model in which the ROS generated by CIN cells is responsible for triggering an immune response. While there are likely to be many substrates affected by a ROS signal, the response may be mediated by the release of redox sensitive substrates like HMGB1 that are known ligands for the Toll pathway in vertebrates; the inflammatory response driven by Toll activation then significantly contributes to CIN cell death.

\section{Cell death caused by CIN is TNFa and JNK dependent}

Toll signaling in Drosophila is known to generate a humoral response through antimicrobial peptides and to activate hemocytes that contribute to tumour clearance by TNF signalling $[14,29]$. We tested whether Eiger, the Drosophila homolog of TNF $\alpha$ was also involved in mediating the apoptosis of CIN cells. Knockdown of TNF $\alpha$ /eiger by dsRNA in wing discs significantly reduced the apoptosis in CIN cells (Figure 4b). TNFa has been shown to cause cell death via the JNK pathway [30], so we tested the role of JNK in mediating the response to CIN. Knockdown of JNK strongly rescued the apoptosis of these CIN cells (Figure 4c). Looking downstream of JNK, we found that the JNK effector Mmp1 [31] was elevated in CIN cells (Figure 5b) but was lost if Toll signalling was reduced (Figure 5c). Overexpression of either TNF $\alpha$ or the Toll effector NFkB/Dorsal was sufficient to give elevated Mmp1 levels in normal wing discs (Figure 5e and Figure 5f), consistent with JNK and Mmpl activation being downstream of Toll signaling. Our results show that Toll/ $\mathrm{NF} \kappa \mathrm{B}$ signalling is needed in the CIN tissue itself for the TNF $\alpha$-JNK mediated cell death usually seen when CIN is induced by rad 21 knockdown.

\section{A local immune response is critical for hemocyte recruitment}

One effect of activating the Drosophila innate immune response is the production and recruitment of hemocytes to sites of damage $[32,33]$. Dysplastic or pretumorous tissue in flies can trigger this response, leading to increased numbers of hemocytes and recruitment of hemocytes to the surface of the abnormal tissue [14, 34]. We found that induction of CIN in otherwise normal, nondysplastic tissue also increased the number of hemocytes recruited to the wing discs (Figure 6a-6b, 6d). Simulating local immune activation by $\mathrm{NF} \kappa \mathrm{B}$ overexpression was often sufficient to trigger the JNK-Mmp1 pathway and to recruit hemocytes (Figure 6e-6g). On the other hand, blocking local immune activation by Toll knockdown
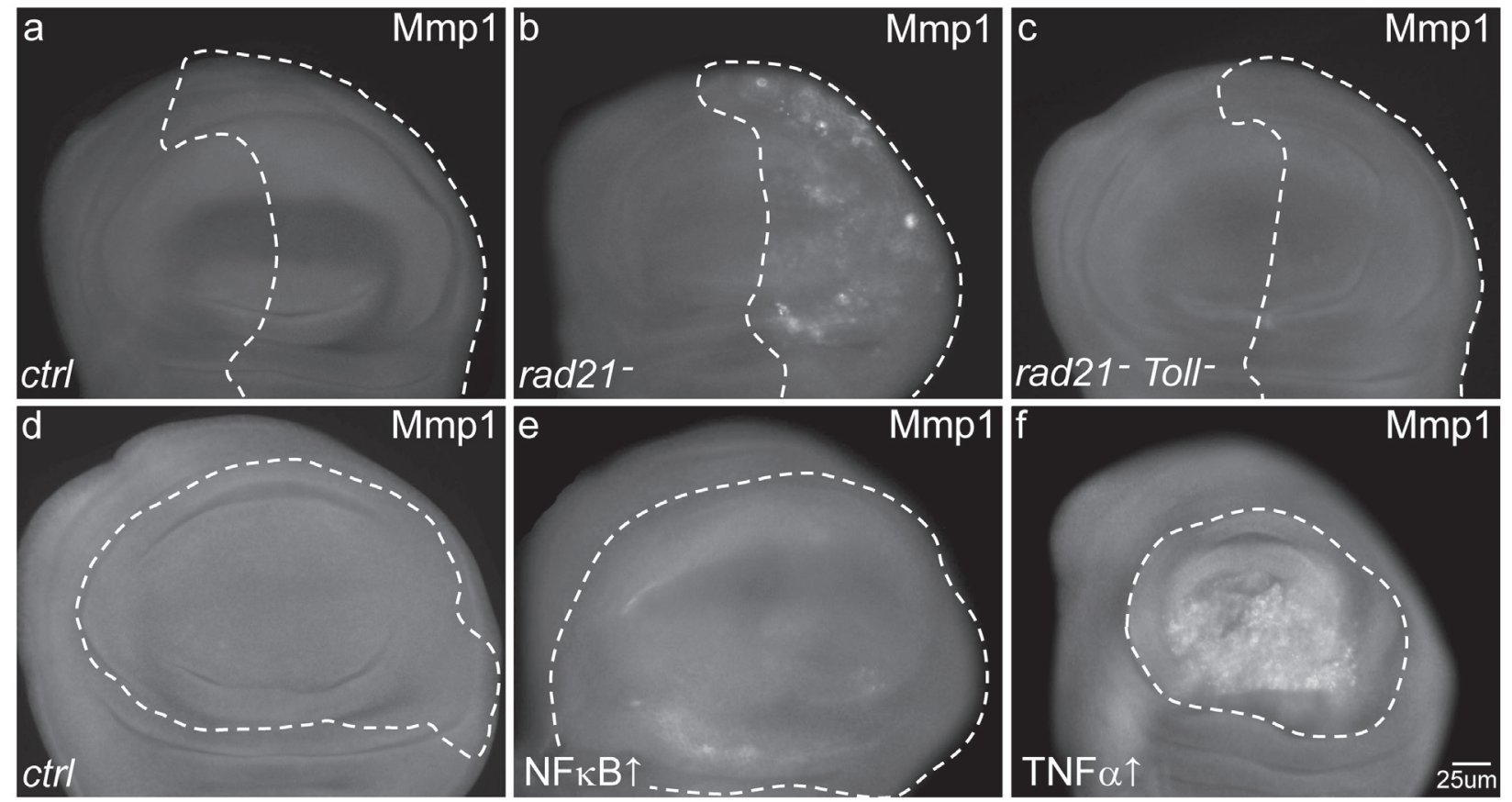

Figure 5: The effects of CIN and local immune signalling on the activation of matrix metalloprotease 1. Anti-Mmp1 antibodies were used to detect the levels of matrix metalloprotease 1 in third instar larval wing discs in which genes were knocked down (dotted regions) using engrailed (a-c) or MS1096 (d-f) drivers. Normal wing discs (a, d) show low levels of Mmp1 staining. (b) When $\mathrm{CIN}$ was induced in the posterior region (dotted) of the disc (engrailed $>$ Gal4, UAS-rad2 $1^{\mathrm{RNAi}}$ UAS-Dicer2), cells showed increased Mmp1 staining. Local signalling via Toll was needed for this effect, as Toll knockdown in these cells (c) reduced the level of Mmp1 staining in CIN cells. Overexpression of NFKB (dorsal) in the wing pouch (e, dotted region) led to a slightly increased level of Mmp1 staining. Overexpression of TNF $\alpha$ (eiger) in the same region (f) gave very high levels of Mmp1. MS1096> Gal4 was used in these overexpression experiments to avoid lethality. 

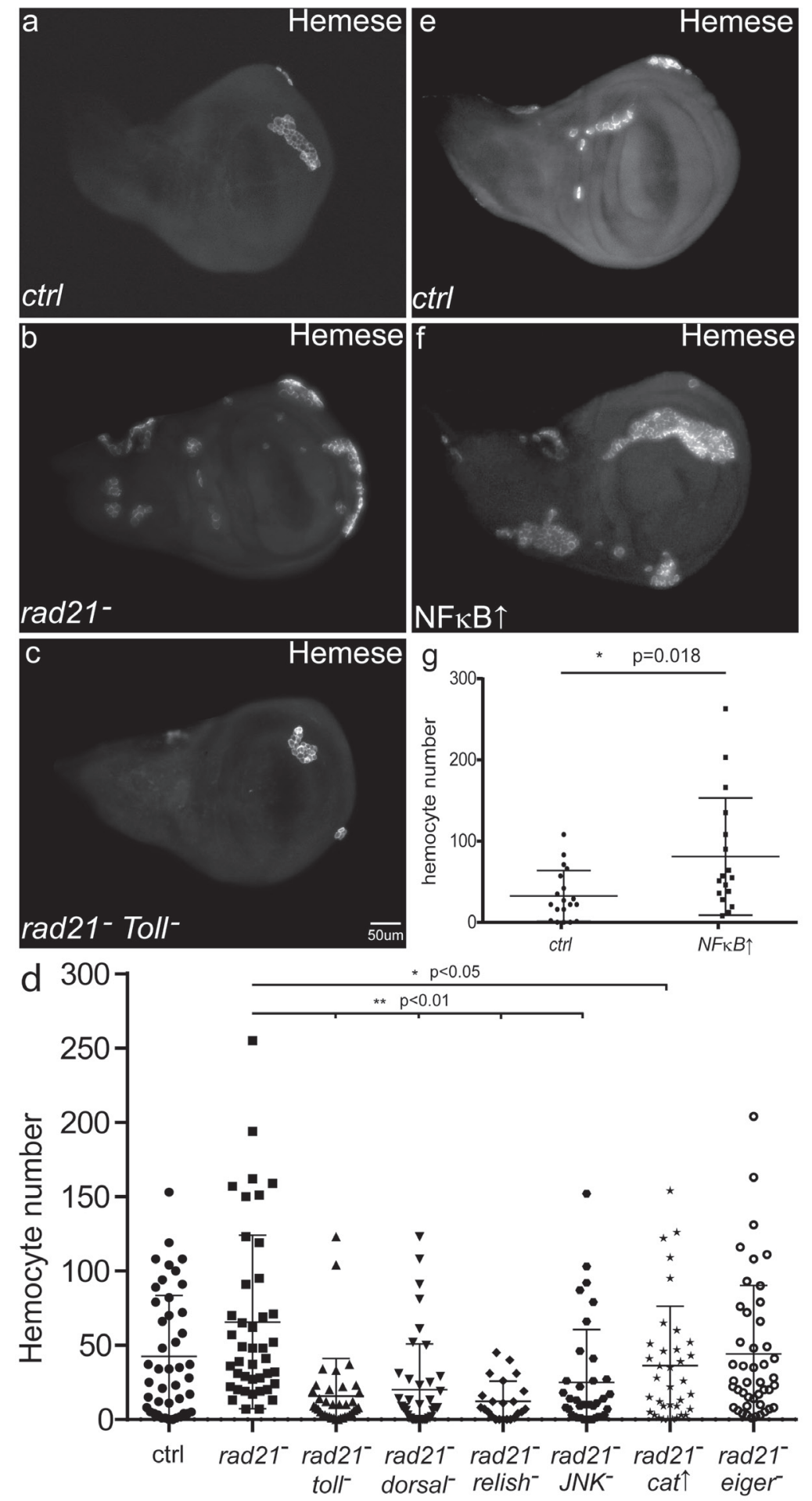

Figure 6: The effects of CIN and immune signalling on the recruitment of hemocytes. Anti-Hemese staining was used to visualize the recruitment of macrophage-like hemocytes to the surface of third instar larval wing discs. When CIN was induced in the wing discs (engrailed $>$ Gal4, UAS-rad2 $1^{\mathrm{RNAi}} U A S$-Dicer 2 ), significantly more hemocytes were recruited (b, $\left.\mathbf{d}, p<0.05\right)$ than to wild type wing discs (a, d). Blocking immune signalling in the CIN cells by Toll knockdown (c, d) greatly reduced the number of hemocytes recruited. (d, g) The quantifications show the number of hemocytes recruited to the wing discs, $\mathrm{n} \geq 20$ in all cases, the error bars show $95 \%$ confidence intervals around the mean. (d) Knockdown of Toll, NFкB homologs (dorsal or relish), or JNK (bsk) strongly reduced the number of hemocytes recruited to CIN wing discs ( $p<0.01$ for each). Overexpression of Catalase to reduce the level of oxidative stress generated by CIN cells also significantly reduced the number of hemocytes recruited to CIN wing discs $(p<0.05)$. Knockdown of eiger (TNF $\alpha)$ did not have a strong effect on the number of hemocytes recruited to CIN wing discs $(p=0.06)$. Simulation of local immune signalling by overexpression of NFKB (MS1096>Gal4, UAS-dorsal) in wing discs (f, g) was sufficient to significantly increase the number of hemocytes recruited compared to control discs $(\mathbf{e}, \mathbf{g})$. All p values were calculated by two-tailed t-tests with Welch's correction. 
reduced the level of Mmp1 activation within CIN cells and lowered the number of hemocytes recruited to the wing discs (Figure 5c and 6c-6d). The same loss of hemocyte recruitment was produced by blocking JNK or either NFkB homolog, or by decreasing ROS levels (Figure $6 \mathrm{~d})$. These results suggest that a ROS-triggered local immune response in the wing disc is critical for hemocyte recruitment and the effective killing of CIN cells (Figure 7).

\section{DISCUSSION}

As a feature of most human solid tumours, chromosomal instability (CIN) has been associated with the initiation of tumorigenesis [4], the development of drug resistance, and the poor prognosis of cancer patients after chemotherapy [35-38]. However, the induction of CIN in proliferating cells is usually detrimental or lethal, and the mechanisms by which cancer cells can tolerate CIN are poorly understood [39]. In order to investigate the signalling pathways that allow CIN tolerance, we carried out viability screening in Drosophila to select genes whose depletion could rescue lethality caused by CIN. Interestingly, five of the candidate genes obtained were from the Drosophila immune system. Further experiments showed that the depletion of several key genes in Drosophila immune pathways, such as Toll, dorsal and relish, could rescue the apoptosis phenotype caused by CIN in a proliferating tissue (Figure 2). These results strongly suggest that the innate immune system is normally induced to kill CIN cells.

Immune systems have long been thought to be involved in tumorigenesis [7, 40]. Chronic inflammation is thought to contribute to the initiation, promotion and progression of tumours [40]. However, the innate and adaptive immune systems may be able to eliminate transformed cells, so evasion of this immunosurveillance has been recognised as a hallmark of cancer [12, 41]. In this study, we showed that the induction of CIN in Drosophila larvae could trigger the production of antimicrobial peptides from the fat body, the main organ that drives humoral and cellular responses to damage and infection [24, 42]. This result is consistent with recent studies showing that induced tissue overgrowth in flies activates a systemic immune response $[14,42]$. They also saw activation of the Toll pathway in the fat body, however they did not test the role of local immune signalling within the induced tumour.

In this study, we found that induction of CIN not only activated the immune organs, but also triggered an immune response within the proliferating CIN tissue (Figure S3). The Toll and Imd pathways are activated in CIN wing discs and depletion of either Toll or Dorsal just in the wing cells reduced the amount of apoptosis in response to CIN (Figure 2). Our data suggests a model in which the local immune response activates JNK and Mmp1 to recruit hemocytes which in turn trigger apoptosis in those cells (Figure 5 and 6). Consistent with this model, we have seen that enhancing the local immune response by dorsal overexpression greatly increased the level of apoptosis in CIN cells (Figure 2). On the other hand,

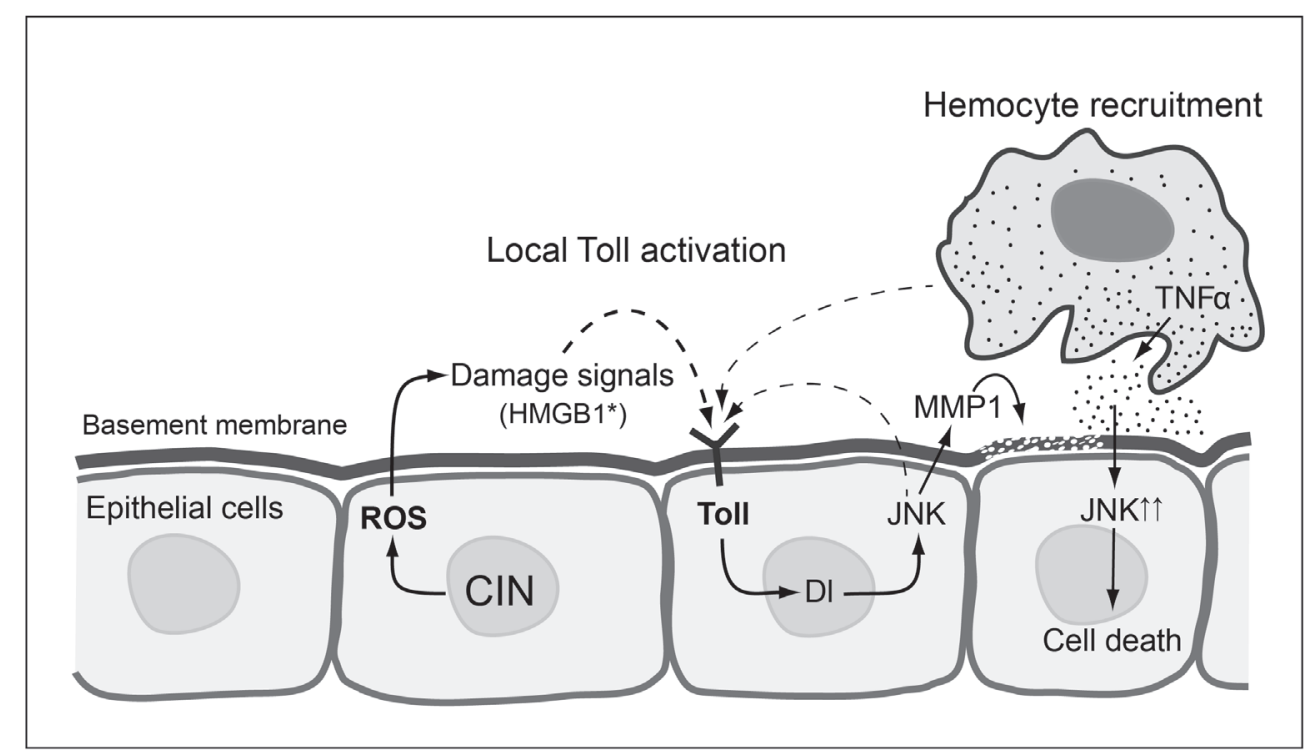

Figure 7: Proposed model for local Toll pathway activation giving apoptosis in response to CIN. CIN cells generate ROS and DAMPs like oxidised HMGB1, which trigger the local immune response in nearby cells through the Toll/NFkB pathway which in turn activates JNK and Mmp1. The activation of Mmp1 leads to basement membrane digestion and hemocyte recruitment. The recruited hemocytes subsequently trigger the apoptosis of underlying cells through secretion of TNF $\alpha$ and further activation of JNK. Note that the signalling processes are drawn in adjacent cells for clarity, but may all occur in the same cell. Dashed lines indicate the production of activated Toll ligand by a process that is not well characterized. This model is informed by data from [34] and [14] on hemocyte responses and [65] on JNK activation of Toll. 
blocking the local immune response and its downstream effectors by knocking down Toll, Dorsal, Relish or JNK could significantly reduce the number of hemocytes recruited (Figure 6d). Hemocytes have been shown to secrete TNF $\alpha$ onto the underlying tissue as well as being a source of the Toll ligand Spz [14], so hemocyte recruitment appears to be a positive feedback loop by which damaged cells attract a source of signals to ensure their own demise (Figure 7).

The activation of local immune signaling in proliferating tissue has been shown to remove slow growing cells [43]. In that case the trigger(s) and targeting are not known, and the Toll receptor itself and JNK had little effect. Thus, although cell competition presents an interesting parallel, it appears to induce a response different to that seen in CIN cells. Local as well as systemic immune responses are also seen in response to infection by bacterial pathogens [44], suggesting that the systemic response alone is either insufficient or requires targeting. For example, bacterial pathogen elimination in infection requires local IMD/Relish pathway activation [45-47]. At least in the gut, it appears that tissue damage and ROS production are used as an additional trigger to improve immune responses to pathogens [44]. In the case of CIN, we think that ROS are made in the mitochondria [16] rather than at the plasma membrane by Duox [48], but the immune effects may be similar.

Toll and Toll-like receptors (TLRs) are the critical mediators of innate immune responses in Drosophila and mammals [23, 49]. Apart from external pathogens, many endogenous molecules released from damaged cells, referred to as DAMPs (damage-associated molecular patterns), can also activate Toll-like receptors [6]. Toll has recently been implicated in the response to tissue dysplasia and damage [14, 50], suggesting a similarity to vertebrate DAMP receptor TLRs. However it should be noted that TLRs frequently detect the DAMP directly, while activation of the Toll ligand Spz is likely to require several intermediate steps [51]. Our results showed that CIN cells exhibit dysfunctional mitochondria and oxidative stress, both of which are known to activate innate immune responses through TLRs [27, 52]. Reducing oxidative stress by over-expressing the antioxidant Catalase significantly rescued the apoptosis phenotype of CIN cells. Consistent with this model in which ROS triggers an immune response, Catalase overexpression also significantly reduced the number of hemocytes recruited (Figure 6d). In addition, removing dysfunctional mitochondria by over-expressing the mitophagy gene park1, which mediates the clearance of abnormal mitochondria [53], also rescues the apoptosis phenotype of CIN cells (our unpublished data). It is not known how CIN generates stressed mitochondria, but current models for stable aneuploidy suggest that altered stoichiometry of proteins can lead to saturation of the protein folding and degradation machinery, leading to ER stress and subsequent mitochondrial stress [54]. We are confident that ROS are an upstream trigger rather than a downstream consequence of apoptosis, because decreasing ROS levels reduced apoptosis in CIN tissue (Figure 3b) and we were able to almost completely block apoptosis without reducing ROS levels (Figure S4).

In order to identify potential mediators of the ROS signal, we tested HMGB1 (high mobility group box 1), which is one of the most intensively studied DAMP molecules. HMGB1 is redox state sensitive and can be released from oxidatively damaged cells, triggering immune responses by binding to Toll-like receptors [27]. We found that HMGB1 depletion could rescue the apoptosis phenotype of CIN cells, suggesting a model in which ROS triggers a local immune response by releasing oxidised HMGB1, leading eventually to CIN cell apoptosis. Consistent with this model, research in mammals has shown that HMGB1 released from dying cells triggers a TLR4 dependent immune response that affects the outcome of traditional cancer therapy [55].

We expected that apoptosis in response to CIN would be mediated by the TNF $/$ /Eiger-JNK pathway, which has been well documented to trigger apoptosis in flies in response to a number of stimuli $[30,56,57]$. Eiger is the Drosophila ortholog of Tumour Necrosis Factor alpha (TNF $\alpha)$ which acts as tumour suppressor and typically drives apoptosis by activation of the intrinsic death pathway though JNK $[30,56]$. JNK signalling has been shown to be dysregulated in several fly "pretumour" models with varying effects including apoptosis, migration, proliferation and DNA repair. $[14,17,31,57$, 58]. In CIN cells, we detected increased JNK signalling (Figure 5), and depletion of either eiger or JNK by RNAi could significantly rescue the apoptosis phenotype (Figure $4)$. These results are consistent with the role of the TNF $\alpha$ JNK pathway as a tumour suppressor signal to eliminate CIN cells by triggering apoptosis. JNK activation would also be expected to promote hemocyte proliferation to increase the local TNF $\alpha$ signal, as observed in response to tissue overgrowth $[14,34]$. JNK signalling through Mmp1 can also lead to invasive cell migration $[31,59]$ typically when apoptosis has been blocked by strong growth factor signalling. Despite the activation of Mmp1 in CIN cells, we have not observed any invasion or metastasis. We speculate that this could be due to apoptotic clearance and the local immune response restraining the invasiveness of CIN cells.

Based on our results, we have hypothesised that CIN cells produce dysfunctional mitochondria and oxidative stress; the generation of ROS and DAMPs such as HMGB1 then triggers a local immune response. This involves signalling through Toll to give JNK activation, which is known to generate signals that attract [34] and expand [14] the hemocyte population. The recruited hemocytes then promote the death of CIN cells through $\mathrm{TNF} \alpha-\mathrm{JNK}$ signalling as well as secreting Spz to increase 
Toll signaling (Figure 7). We also saw some reduction in cell death when TNF $\alpha$ was depleted just in the CIN cells, so we speculate that ROS can generate some local production of $\mathrm{TNF} \alpha$, as has been reported for eye discs [60]. However this more immediate route to cell death (ROS-TNF $\alpha$-JNK) does not appear to be very effective in CIN cells, because without Toll and the involvement of an immune response to amplify the JNK signal, we saw very little CIN cell death (Figure 2).

Our results raise the question of whether such an anti-CIN immune response has clinical implications. Investigation of the innate immune system as a cancer treatment has been going on for more than a century. In the 1890s, Coley injected live bacterial cultures into cancer patients as a treatment to provoke the immune system with some success in treating certain cancer types such as soft tissue sarcoma and lymphoma [61]. Since the 1950s, bacteria-derived materials like polysaccharide have been investigated for cancer immunotherapy. Although the detailed mechanism of their anti-cancer effect is unclear, some of them have been approved for clinical use [6]. DNA damage or DNA repair mutations are known to provoke an innate immune response [41, 62], a response that is likely to also be seen in CIN cells, as they generate ongoing DNA stress $[2,63]$. Our results have suggested that CIN cells are aberrant in a number of significant ways including glucose metabolism, mitochondrial output, ROS levels, JNK signalling, and DNA damage [15-17], and that some or all of these contribute to a signal that generates the local and systemic immune responses needed to eliminate the damaged cells. It remains to be seen to what extent this response can be exploited therapeutically.

\section{MATERIALS AND METHODS}

\section{Drosophila stocks}

The fly stocks used in this paper are as follows: mad2-RNAi (VDRC 47918), Rad21-RNAi (Bloomington \#36786), Eiger (TNFa)-RNAi (VDRC108814), UAScatalase (Bloomington \#24621), HMGB1-RNAi (Bloomington \#31960), Drosomycin-GFP [24], UAS-p35 (Bloomington \#5073).

Driver stocks: daughterless ( $d a)$-Gal4 for ubiquitous expression, engrailed (en)-Gal4 for gene expression in the posterior region of wing discs and MS1096-Gal4 for wing pouch expression, all from Bloomington Drosophila stock centre.

\section{Viability screening}

Candidate genes were knocked down in the CIN background (mad2 knockdown) to see their effect on the viability of CIN flies: UAS $>\operatorname{mad} 2 \mathrm{RNAi} / \mathrm{CyO}$; $d a>\mathrm{Gal} 4 /$
TM6 tubulin $>$ Gal80ts $\times$ UAS $>$ candidate-RNAi. The crosses were set at $30^{\circ} \mathrm{C}$ which was lethal for CIN flies crossed to negative controls.

\section{RNA purification and quantitative real-time PCR (qPCR) assays}

Five third instar larvae from each genotype (in triplicate for each genotype) were chosen and washed in PBS and were quickly transferred and homogenised in cold Trizol reagent on ice and then stored at $-80^{\circ} \mathrm{C}$ before processing as described [15]. Primers pairs used in this paper:

$\operatorname{mad} 2 \quad$ F/R:GGCGACCAAAAACTGCATCA/
GGTAAATTCCGCGTTGGAAGA
rp49 F/R:ATCGATATGCTAAGCTGTCGCAC/
TGTCGATACCCTTGGGCTTG

\section{Karyotype analysis}

For measuring the level of aneuploidy, wing discs from third instar larvae were dissected out in PBS, and were incubated for $10 \mathrm{~min}$ in $0.5 \%$ sodium citrate solution. Then these discs were treated with $45 \%$ acetic acid for 2 min and $60 \%$ acetic acid for $1 \mathrm{~min}$ on a cover slip. Treated wing discs were squashed quickly between a coverslip and a slide and placed into liquid nitrogen. The cover slip was removed and the squashed discs were stained with Hoechst 33342 for $10 \mathrm{~min}$ and washed with PBST for 20 min before mounted in $80 \%$ glycerol. The karyotypes of different genotypes were compared using $\chi^{2}$ analysis to detect significant variation from the expected proportions of euploid and aneuploid cells.

\section{Cell death}

Acridine Orange (AO) staining was used to measure the cell death in larval wing imaginal discs [15]. Third instar larvae were dissected in PBS for imaginal discs; the collected imaginal discs were incubated in $1 \mathrm{mM}$ AO for 2 mins then transferred to a slide after a brief wash. Then the treated imaginal discs were immediately mounted in PBS with a cover slip on before microscopy. The results of AO were normalized by subtracting the wild type region value from the test region value (eg. engrailed-driven region) as identified by $\mathrm{UAS}>C D 8-G F P$ expression. The background noise of all images was subtracted in ImageJ using a rolling ball radius of 10 pixels.

\section{Oxidative stress assay}

The level of reactive oxygen species (ROS) in CIN cells was measured by using the fluorogenic probe CellROX from Life Technologies. The third instar larvae 
were dissected in D22 media $\mathrm{pH}$ 6.8. Then the dissected imaginal wing discs were transferred into $5 \mu \mathrm{M}$ CellRox (in D22 media) for 15 mins; after this, the wing discs were quickly washed in PBS and fixed in 3.7\% formaldehyde for $5 \mathrm{~min}$ then mounted in $80 \%$ glycerol for imaging.

\section{Mitochondrial stress}

The level of mitochondrial stress in CIN cells was measured by using the fluorogenic probe TMRE from Life Technologies. Third instar larvae were dissected in PBS and transferred into $0.05 \mu \mathrm{M}$ TMRE solution for $10 \mathrm{mins}$ incubation and then washed in PBS for 10 mins. Then the treated imaginal discs were immediately mounted in PBS for imaging.

\section{Immunostaining}

Immunostaining was used on dissected wing imaginal discs for different purposes. Third instar larvae were dissected in PBS for imaginal discs; the collected imaginal discs were fixed in $3.7 \%$ formaldehyde for 20 mins and then wash for 30 mins in $0.2 \%$ PBST ( $1 \mathrm{xPBS}+0.2 \%$ Tween). For anti-hemese staining, the fixation time was $4^{\circ} \mathrm{C}$ overnight, with no shaking through all the process. The fixed imaginal wing discs were then blocked in PBSTF $(1 \mathrm{xPBS}+0.2 \%$ Tween $+5 \%$ fetal calf serum) for 30 mins and stained with the primary antibody for $2.5 \mathrm{hrs}$ (at room temperature) or overnight (at $4^{\circ} \mathrm{C}$ ). After staining with the primary antibody, the wing discs were washed in PBSTF for 30 mins then transferred to a secondary antibody solution for $2.5 \mathrm{hrs}$ at room temperature in the dark. After 30 mins washing in PBST, the wing discs were mounted in $80 \%$ glycerol-PBS.

The source and concentration of antibodies used in this paper are as follows: Rabbit anti-caspase 3 (D175, 1: 100) from Cell Signalling; mouse anti-dorsal (7A4, $22 \mu \mathrm{g} /$ $\mathrm{ml})$ and mouse anti-MMP1 (14A3D2, $5.3 \mu \mathrm{g} / \mathrm{ml})$ from the Developmental Studies Hybridoma Bank; mouse antihemese $(1.5 \mu \mathrm{g} / \mathrm{ml})$ [64].

The secondary antibodies used were CY3 anti-rabbit (1: 100), rhodamine anti-mouse (1: 200).

\section{Data analysis}

All microscopy was done on a Zeiss Axioplan2 microscope. Axiovision software (Carl Zeiss), Adobe Photoshop, Adobe Illustrator and ImageJ were used for image processing and quantification. Statistical analysis was carried out in GraphPad Prism using either t-tests or $\chi^{2}$ tests as indicated.

\section{ACKNOWLEDGMENTS}

This work was supported by grants from the NHMRC to RBS and SLG (1087308 and 1027878) and a CSC scholarship for DL. We thank E. Genockey for technical assistance, L. O'Keefe for helpful comments on the manuscript and T. Shandala for providing stocks.

\section{CONFLICTS OF INTEREST}

The authors declare no conflict of interest.

\section{REFERENCES}

1. Thompson SL, Bakhoum SF and Compton DA. Mechanisms of chromosomal instability. Current biology : CB. 2010; 20:R285-295.

2. Burrell RA, McClelland SE, Endesfelder D, Groth P, Weller MC, Shaikh N, Domingo E, Kanu N, Dewhurst SM, Gronroos E, Chew SK, Rowan AJ, Schenk A, Sheffer M, Howell M, Kschischo M, et al. Replication stress links structural and numerical cancer chromosomal instability. Nature. 2013; 494:492-496.

3. McGranahan N, Burrell RA, Endesfelder D, Novelli MR and Swanton C. Cancer chromosomal instability: therapeutic and diagnostic challenges. EMBO reports. 2012; 13:528-538.

4. Nowak MA, Komarova NL, Sengupta A, Jallepalli PV, Shih Ie M, Vogelstein B and Lengauer C. The role of chromosomal instability in tumor initiation. Proceedings of the National Academy of Sciences of the United States of America. 2002; 99:16226-16231.

5. Gerlinger $M$ and Swanton C. How Darwinian models inform therapeutic failure initiated by clonal heterogeneity in cancer medicine. British journal of cancer. 2010; 103:1139-1143.

6. Maruyama K, Selmani Z, Ishii H and Yamaguchi K. Innate immunity and cancer therapy. International immunopharmacology. 2011; 11:350-357.

7. Grivennikov SI, Greten FR and Karin M. Immunity, inflammation, and cancer. Cell. 2010; 140(6):883-899.

8. Shankaran V, Ikeda H, Bruce AT, White JM, Swanson PE, Old LJ and Schreiber RD. IFNgamma and lymphocytes prevent primary tumour development and shape tumour immunogenicity. Nature. 2001; 410:1107-1111.

9. Kim R, Emi M and Tanabe K. Cancer immunoediting from immune surveillance to immune escape. Immunology. 2007; 121:1-14.

10. Dunn GP, Old LJ and Schreiber RD. The immunobiology of cancer immunosurveillance and immunoediting. Immunity. 2004; 21:137-148.

11. Nelson BH. The impact of T-cell immunity on ovarian cancer outcomes. Immunological reviews. 2008; 222:101116. 
12. Hanahan D and Weinberg RA. Hallmarks of cancer: the next generation. Cell. 2011; 144:646-674.

13. Ermolaeva MA, Segref A, Dakhovnik A, Ou HL, Schneider JI, Utermohlen O, Hoppe $\mathrm{T}$ and Schumacher B. DNA damage in germ cells induces an innate immune response that triggers systemic stress resistance. Nature. 2013; 501:416-420.

14. Parisi F, Stefanatos RK, Strathdee K, Yu Y and Vidal M. Transformed epithelia trigger non-tissue-autonomous tumor suppressor response by adipocytes via activation of Toll and Eiger/TNF signaling. Cell reports. 2014; 6:855-867.

15. Shaukat Z, Wong HW, Nicolson S, Saint RB and Gregory SL. A screen for selective killing of cells with chromosomal instability induced by a spindle checkpoint defect. PloS one. 2012; 7:e47447.

16. Shaukat Z, Liu D, Choo A, Hussain R, O'Keefe L, Richards R, Saint R and Gregory SL. Chromosomal instability causes sensitivity to metabolic stress. Oncogene. 2014; DOI: 10.1038/onc.2014.344.

17. Wong HW, Shaukat Z, Wang J, Saint R and Gregory SL. JNK signaling is needed to tolerate chromosomal instability. Cell Cycle. 2014; 13:622-631.

18. Cucco F, Servadio A, Gatti V, Bianchi P, Mannini L, Prodosmo A, De Vitis E, Basso G, Friuli A, Laghi L, Soddu S, Fontanini G and Musio A. Mutant cohesin drives chromosomal instability in early colorectal adenomas. Human molecular genetics. 2014; DOI: 10.1093/hmg/ ddu394.

19. Vass S, Cotterill S, Valdeolmillos AM, Barbero JL, Lin E, Warren WD and Heck MM. Depletion of Drad21/Scc1 in Drosophila cells leads to instability of the cohesin complex and disruption of mitotic progression. Current biology : CB. 2003; 13:208-218.

20. Xu H, Yan Y, Deb S, Rangasamy D, Germann M, Malaterre J, Eder NC, Ward RL, Hawkins NJ, Tothill RW, Chen L, Mortensen NJ, Fox SB, McKay MJ and Ramsay RG. Cohesin Rad21 mediates loss of heterozygosity and is upregulated via Wnt promoting transcriptional dysregulation in gastrointestinal tumors. Cell reports. 2014; 9:1781-1797.

21. Morais da Silva S, Moutinho-Santos T and Sunkel CE. A tumor suppressor role of the Bub3 spindle checkpoint protein after apoptosis inhibition. The Journal of cell biology. 2013; 201:385-393.

22. Hoffmann JA. The immune response of Drosophila. Nature. 2003; 426:33-38.

23. Valanne S, Wang JH and Ramet M. The Drosophila Toll signaling pathway. Journal of immunology. 2011; 186:649656.

24. Ferrandon D, Jung AC, Criqui M, Lemaitre B, UttenweilerJoseph S, Michaut L, Reichhart J and Hoffmann JA. A drosomycin-GFP reporter transgene reveals a local immune response in Drosophila that is not dependent on the Toll pathway. The EMBO journal. 1998; 17:1217-1227.
25. Ferrandon D, Imler JL, Hetru C and Hoffmann JA. The Drosophila systemic immune response: sensing and signalling during bacterial and fungal infections. Nature reviews Immunology. 2007; 7:862-874.

26. Hay BA, Wolff T and Rubin GM. Expression of baculovirus P35 prevents cell death in Drosophila. Development. 1994; 120:2121-2129.

27. Lugrin J, Rosenblatt-Velin N, Parapanov R and Liaudet L. The role of oxidative stress during inflammatory processes. Biological chemistry. 2014; 395:203-230.

28. Apetoh L, Ghiringhelli F, Tesniere A, Criollo A, Ortiz C, Lidereau R, Mariette C, Chaput N, Mira JP, Delaloge $\mathrm{S}$, Andre F, Tursz T, Kroemer G and Zitvogel L. The interaction between HMGB1 and TLR4 dictates the outcome of anticancer chemotherapy and radiotherapy. Immunological reviews. 2007; 220:47-59.

29. Schmid MR, Anderl I, Vesala L, Vanha-aho LM, Deng XJ, Ramet M and Hultmark D. Control of Drosophila blood cell activation via Toll signaling in the fat body. PloS one. 2014; 9:e102568.

30. Moreno E, Yan M and Basler K. Evolution of TNF signaling mechanisms: JNK-dependent apoptosis triggered by Eiger, the Drosophila homolog of the TNF superfamily. Current biology : CB. 2002; 12:1263-1268.

31. Uhlirova $\mathrm{M}$ and Bohmann D. JNK- and Fos-regulated Mmp1 expression cooperates with Ras to induce invasive tumors in Drosophila. The EMBO journal. 2006; 25:52945304.

32. Babcock DT, Brock AR, Fish GS, Wang Y, Perrin L, Krasnow MA and Galko MJ. Circulating blood cells function as a surveillance system for damaged tissue in Drosophila larvae. Proceedings of the National Academy of Sciences of the United States of America. 2008; 105:1001710022.

33. Krautz R, Arefin B and Theopold U. Damage signals in the insect immune response. Frontiers in plant science. 2014; DOI: $10.3389 /$ fpls.2014.00342.

34. Pastor-Pareja JC, $\mathrm{Wu} \mathrm{M}$ and $\mathrm{Xu} \mathrm{T}$. An innate immune response of blood cells to tumors and tissue damage in Drosophila. Disease models \& mechanisms. 2008; 1:144154.

35. Swanton C, Nicke B, Schuett M, Eklund AC, Ng C, Li Q, Hardcastle T, Lee A, Roy R, East P, Kschischo M, Endesfelder D, Wylie P, Kim SN, Chen JG, Howell M, et al. Chromosomal instability determines taxane response. Proceedings of the National Academy of Sciences of the United States of America. 2009; 106:8671-8676.

36. Lee AJ, Endesfelder D, Rowan AJ, Walther A, Birkbak NJ, Futreal PA, Downward J, Szallasi Z, Tomlinson IP, Howell M, Kschischo M and Swanton C. Chromosomal instability confers intrinsic multidrug resistance. Cancer research. 2011; 71:1858-1870.

37. Walther A, Houlston $\mathrm{R}$ and Tomlinson I. Association between chromosomal instability and prognosis in 
colorectal cancer: a meta-analysis. Gut. 2008; 57:941-950.

38. Watanabe T, Kobunai T, Yamamoto Y, Matsuda K, Ishihara S, Nozawa K, Yamada H, Hayama T, Inoue E, Tamura J, Iinuma H, Akiyoshi T and Muto T. Chromosomal instability (CIN) phenotype, CIN high or CIN low, predicts survival for colorectal cancer. Journal of clinical oncology : official journal of the American Society of Clinical Oncology. 2012; 30:2256-2264.

39. Gordon DJ, Resio B and Pellman D. Causes and consequences of aneuploidy in cancer. Nature reviews Genetics. 2012; 13:189-203.

40. Karin M and Greten FR. NF-kappaB: linking inflammation and immunity to cancer development and progression. Nature reviews Immunology. 2005; 5:749-759.

41. Vesely MD, Kershaw MH, Schreiber RD and Smyth MJ. Natural innate and adaptive immunity to cancer. Annual review of immunology. 2011; 29:235-271.

42. Hauling T, Krautz R, Markus R, Volkenhoff A, Kucerova $\mathrm{L}$ and Theopold U. A Drosophila immune response against Ras-induced overgrowth. Biology open. 2014; 3:250-260.

43. Meyer SN, Amoyel M, Bergantinos C, de la Cova C, Schertel C, Basler K and Johnston LA. An ancient defense system eliminates unfit cells from developing tissues during cell competition. Science. 2014; 346:1258236.

44. Buchon N, Silverman N and Cherry S. Immunity in Drosophila melanogaster - from microbial recognition to whole-organism physiology. Nature reviews Immunology. 2014; 14:796-810.

45. Nehme NT, Liegeois S, Kele B, Giammarinaro P, Pradel E, Hoffmann JA, Ewbank JJ and Ferrandon D. A model of bacterial intestinal infections in Drosophila melanogaster. PLoS pathogens. 2007; 3:e173.

46. Vodovar N, Vinals M, Liehl P, Basset A, Degrouard J, Spellman P, Boccard F and Lemaitre B. Drosophila host defense after oral infection by an entomopathogenic Pseudomonas species. Proceedings of the National Academy of Sciences of the United States of America. 2005; 102:11414-11419.

47. Tzou P, Ohresser S, Ferrandon D, Capovilla M, Reichhart JM, Lemaitre B, Hoffmann JA and Imler JL. Tissue-specific inducible expression of antimicrobial peptide genes in Drosophila surface epithelia. Immunity. 2000; 13:737-748.

48. Ha EM, Lee KA, Park SH, Kim SH, Nam HJ, Lee HY, Kang D and Lee WJ. Regulation of DUOX by the Galphaqphospholipase Cbeta-Ca2+ pathway in Drosophila gut immunity. Developmental cell. 2009; 16:386-397.

49. Takeda K and Akira S. Toll-like receptors in innate immunity. International immunology. 2005; 17:1-14.

50. Ming M, Obata F, Kuranaga E and Miura M. Persephone/ Spatzle pathogen sensors mediate the activation of Toll receptor signaling in response to endogenous danger signals in apoptosis-deficient Drosophila. J Biol Chem. 2014; 289:7558-7568.

51. Kounatidis I and Ligoxygakis P. Drosophila as a model system to unravel the layers of innate immunity to infection. Open biology. 2012; 2:120075.

52. West AP, Shadel GS and Ghosh S. Mitochondria in innate immune responses. Nature reviews Immunology. 2011; 11:389-402.

53. Narendra D, Tanaka A, Suen DF and Youle RJ. Parkin is recruited selectively to impaired mitochondria and promotes their autophagy. The Journal of cell biology. 2008; 183:795803.

54. Dephoure N, Hwang S, O'Sullivan C, Dodgson SE, Gygi SP, Amon A and Torres EM. Quantitative proteomic analysis reveals posttranslational responses to aneuploidy in yeast. eLife. 2014; 3:e03023.

55. Apetoh L, Ghiringhelli F, Tesniere A, Obeid M, Ortiz C, Criollo A, Mignot G, Maiuri MC, Ullrich E, Saulnier P, Yang H, Amigorena S, Ryffel B, Barrat FJ, Saftig P, Levi F, et al. Toll-like receptor 4-dependent contribution of the immune system to anticancer chemotherapy and radiotherapy. Nature medicine. 2007; 13:1050-1059.

56. Igaki T, Kanda H, Yamamoto-Goto Y, Kanuka H, Kuranaga E, Aigaki T and Miura M. Eiger, a TNF superfamily ligand that triggers the Drosophila JNK pathway. The EMBO journal. 2002; 21:3009-3018.

57. Igaki T, Pastor-Pareja JC, Aonuma H, Miura M and Xu T. Intrinsic tumor suppression and epithelial maintenance by endocytic activation of Eiger/TNF signaling in Drosophila. Developmental cell. 2009; 16:458-465.

58. Brumby AM, Goulding KR, Schlosser T, Loi S, Galea R, Khoo P, Bolden JE, Aigaki T, Humbert PO and Richardson HE. Identification of novel Ras-cooperating oncogenes in Drosophila melanogaster: a RhoGEF/Rho-family/JNK pathway is a central driver of tumorigenesis. Genetics. 2011; 188:105-125.

59. Miles WO, Dyson NJ and Walker JA. Modeling tumor invasion and metastasis in Drosophila. Disease models \& mechanisms. 2011; 4:753-761.

60. Yang G, Parkhurst CN, Hayes S and Gan WB. Peripheral elevation of TNF-alpha leads to early synaptic abnormalities in the mouse somatosensory cortex in experimental autoimmune encephalomyelitis. Proceedings of the National Academy of Sciences of the United States of America. 2013; 110:10306-10311.

61. Coley WB. II. Contribution to the Knowledge of Sarcoma. Annals of surgery. 1891; 14:199-220.

62. Gasser S and Raulet DH. The DNA damage response arouses the immune system. Cancer research. 2006; 66:3959-3962.

63. Janssen A, van der Burg M, Szuhai K, Kops GJ and Medema RH. Chromosome segregation errors as a cause of DNA damage and structural chromosome aberrations. Science. 2011; 333:1895-1898.

64. Csordas G, Varga GI, Honti V, Jankovics F, Kurucz E and Ando I. In vivo immunostaining of hemocyte compartments in Drosophila for live imaging. PloS one. 2014; 9:e98191. 
65. Wu C, Chen C, Dai J, Zhang F, Chen Y, Li W, PastorPareja JC and Xue L. Toll pathway modulates TNF-induced JNK-dependent cell death in Drosophila. Open biology. 2015; 5: DOI: 10.1098/rsob.140171. 